\title{
Research Paper: Investigation of the Lifting Speed on Lumber Muscles Activities
}

\author{
Siavash Etemadinezhad ${ }^{1}$, Farhad Tabatabai Ghomshe ${ }^{2}$, Mohammad Hossein Sorayani Bafghi ${ }^{3 *}$, Jamshid Yazdani Charati ${ }^{1}$
}

1. Health Sciences Research Center, Mazandaran University of Medical Sciences, Sari, Iran.

2. Pediatric Neurorehabilitation Research Center, University of Social Welfare and Rehabilitation Sciences, Tehran, Iran.

3. Department of Ergonomics, Faculty of Public Health, Mazandaran University of Medical Sciences, Sari, Iran.

Citation: Etemadinezhad S, Tabatabai Ghomshe F, Sorayani Bafghi MH, Yazdani Charati J. Investigation of the Lifting Speed on Lumber Muscles Activities. Physical Treatments. 2016; 6(2):103-108. https://doi.org/10.18869/NRIP.PTJ.6.2.103

dol: : https://doi.org/10.18869/NRIP.PTJ.6.2.103

Article info:

Received: 02 Jan. 2016

Accepted: 07 May 2016
Keywords:

Lifting, Squat,

Electromyography,

Lumbar spine, Speed

\begin{abstract}
A B S T R A C T
Purpose: Low back pain is one of the most common musculoskeletal disorders, and lifting is one of its risk factors. The activities of lumbar muscles as the main muscles involved in lifting are important with regard to identification of the allowable limits and the injury mechanisms as well as the motor control aspects. This study aimed to investigate the effects of the lifting speed on the lumbar muscles activities during the lifting of the light loads by squat method.
\end{abstract}

Methods: In this study, 27 healthy men were tested using electromyography (EMG). The data were collected from the erector spinae and the multifidus muscles. The signal processing was performed using MATLAB software after normalization to MVC and the statistical analysis was performed with SPSS 16.

Results: The muscles activities at fast, medium, and slow speeds were not significantly different $(\mathrm{P}>0.05)$; however, the difference between the numerical values of muscles activities at low and high speeds were clearly visible in the diagram. The highest activity was observed in multifidus muscles and then in erector spinae muscles.

Conclusion: The results indicated that the lifting speed does not have significant effect on the activity of lumbar muscles during the lifting of light loads, but it should be noted that it can be a risk factor in the long term for individuals who are lifting a lot of loads in a day.

\section{Introduction}

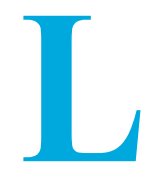

ow back pain (LBP) is a widespread problem, which is considered as one of the most costly musculoskeletal disorders, so that approximately $60 \%$ to $80 \%$ of people experience this problem in their lifetime [1]. Furthermore, other studies reported that more than
$90 \%$ of adults have experienced LBP at least once in their life [2]. Many studies have been conducted on the lifting of loads as one of the risk factors for LBP [3-6].

Some of these biomechanical studies are towards the lifting of loads by squat and stoop methods [7]. The results of a research on the lifting speed showed that the increasing lifting speed rises the forces and the moment

\section{* Corresponding Author:}

Mohammad Hossein Sorayani Bafghi, PhD

Address: Department of Ergonomics, Faculty of Public Health, Mazandaran University of Medical Sciences, Sari, Iran.

Phone: +98 (912) 7254956

E-mail: mhsb128@yahoo.com 
around L5-S1 joint [8]. Another study has also shown that increasing the load rate and the lifting speed rises the amount of bending moment on the lumbar spine [9]. In another research on the effect of reducing the load rate on the kinematic and torque parameters of lifting in the spine, it was observed that the slight increase in load rate is compensated by the lifting speed of the trunk and there was no difference in the load rate exerted on the spine [10].

In another study performed to evaluate the effects of 3 methods of lifting on the lumbar muscles by electromyography (EMG), it was observed that the lifting speed is an effective factor on the differences between the lifting methods [11]. The study of kinematic and kinetic parameters of the load lifting showed that the bending moment increases by rising the speed and vice versa. It is also observed that the motor control system is independent of the variation of the load rate, but it depends on the various lifting speeds [12]. Furthermore, a previous study revealed that the low speed lifting can be dangerous as much as the high speed lifting [13]. In addition, the lifting speed, which is a risk factor for LBP [14-16], is one of the important factors that can affect the electrical activity of the erector spinae muscles [17].

The importance of the lumbar muscles in lifting can be inferred from the previous studies. This study was conducted because little evidence exists on the lifting the light loads by squat method using EMG. This study aimed to evaluate the effect of lifting speed on the electrical activities of the lumbar muscles, including multifidus and erector spinae muscles in healthy individuals.

\section{Materials and Methods}

This was a cross-sectional study and the participants were selected using the convenience sampling among the available individuals in the age range of 20-30 years. The inclusion and exclusion criteria of individuals were observed. All 27 healthy men had no previous history of back pain or neuromuscular problems in the lumbar region confirmed by a specialist. The selected individuals to participate in study had the body mass index between 20 and $25 \mathrm{~kg} / \mathrm{m}^{2}$. The inclusion criteria were all healthy people in order to perform this study among normal and healthy population. Exclusion criteria were having the symptoms of low back or musculoskeletal pain. In addition, the obese individuals with subcutaneous fat, as a factor for high impedance, were excluded from the study to obtain the data from electrodes with lowest voltage drop.

The study participation or leaving was entirely voluntary and with the consent of the participant. This study was ap-

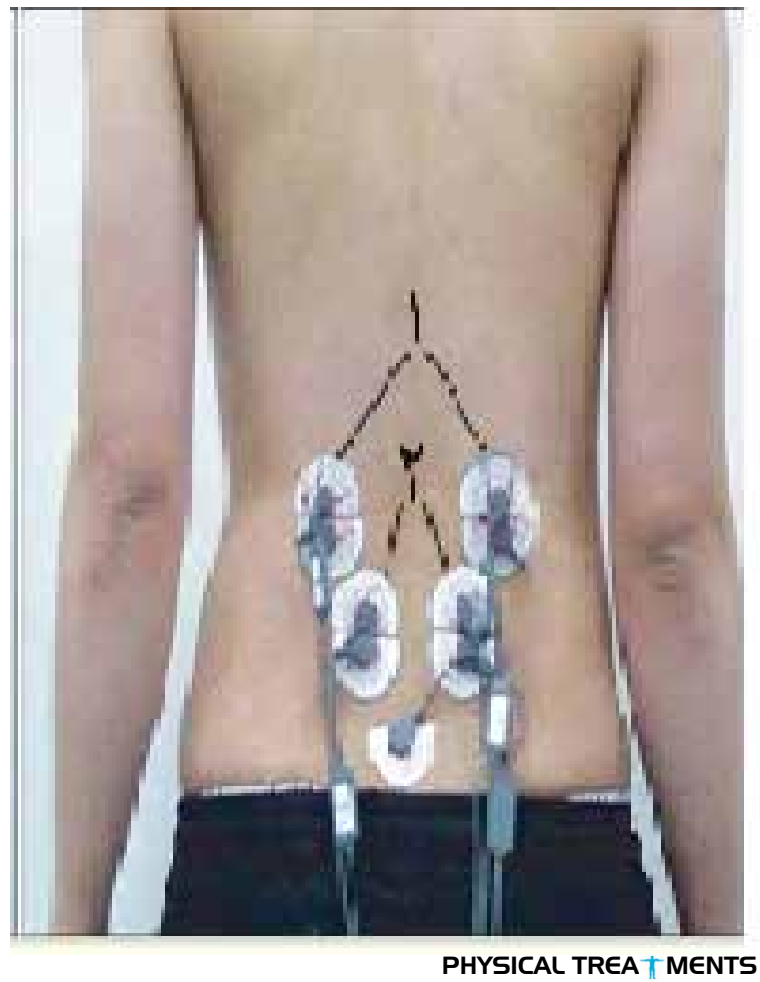

Figure 1. Locations of the electrodes (1: Erector spinae, 2: Multifidus)

proved by the Ethics Committee of Mazandaran University of Medical Sciences. A Biometrics 8-channel electromyography device was used to record the muscle activity in the lumbar extensor and flexor muscles of the trunk

This 8-channel EMG device was used to record the activity of the trunk extensors (erector spinae and multifidus muscles) during the lifting. After skin preparation, which was performed with complete shaving of the location of the electrodes with a sharp blade and cleaning of the skin with cotton and alcohol, the surface electrodes were placed on both side of the body over the left and right of the erector spinae and multifidus muscles.

\section{EMG electrodes locations}

The erector spinae electrode location should be placed 1 finger width medial to the line from the posterior spina iliaca superior to the lowest point of the lower rib, at the level of L2. The multifidus electrode location should be placed on and aligned with a line from caudal tip posterior spina iliaca superior to the interspace between L1 and L2 interspace at the level of L5 spinous process (about $2-3 \mathrm{~cm}$ from the midline).

The locations of the electrodes were determined according to SENIAM [18], which is shown in Figure 1. In the trial protocol, the test procedure was taught to all 
participants prior to the study and they were asked to lift a light weight with three speeds: slow, medium and fast by squat method (kneeling down and without bending the lower back). The weight distribution was even and located within a simple box with average size. After collecting the data, the signal processing was carried out using MATLAB 2014. Followed by the elimination of the noise and filtering the signal, electrical activities of the muscles were normalized with respect to their maximum voluntary contraction. Finally, statistical outputs were achieved using SPSS16.

\section{Results}

After obtaining EMG data and signal processing, the muscle activity level was analyzed using mean variable. EMG outputs have different results and different interpretations. In this study on muscles activities, the mean muscles activities was used. The mean activities of the left and right erector spinae and the multifidus muscles and their standard deviations are presented in Table 1. The statistical analysis of the data was carried out using multivariate test. The results for the left and right multifidus and erector spinae muscles were obtained according to the variation of the lifting speed. It should be noted that the muscle activity is the dependent variable and the lifting speed is the independent one. The results are presented in Tables 1 and 2 and Figure 2.
Since this study aimed to evaluate the effect of the speed on the average muscles activities (low, medium and fast), the multivariate test was applied. The statistical analyses indicated that the $\mathrm{P}$ value is $0.461(\mathrm{P}>0.05)$ in Hoteling's trace so there was no significant correlation. To understand the effect of speed on each muscle, the beta number in Table 2, which is related to the parameter estimates, should be considered ( $\mathrm{B}$ indicate the effect of speed on each muscle).

The lowest $\mathrm{P}$ value was related to the comparison between the muscle activities in the slow and fast speeds. It should be noted that the abbreviation of the words of "slow", "medium", and "fast" was shown with "S", "M", and "F", respectively.

\section{Discussion}

Although no significant association was seen between the speed and muscle activity, the muscles activity levels were higher in fast speeds compared to the slow ones (Figure 2). The difference in values between the medium and fast speeds was negligible. According to Houtman et al. [14], who studied the psychological stressors and musculoskeletal problems, the speed of working was a risk factor for LBP and musculoskeletal problems when this speed causes the psychological stress for the individuals. Also, it is not logical to understand the impact of the speed level on the muscle activity because the re-

Table 1. Descriptive characteristics of muscles and movement speed.

\begin{tabular}{|c|c|c|c|}
\hline Muscle & Speed & Mean & SD \\
\hline \multirow{3}{*}{ MF r } & Fast & 0.20308 & 0.080284 \\
\hline & Medium & 0.16510 & 0.077679 \\
\hline & Slow & 0.15439 & 0.082415 \\
\hline \multirow{3}{*}{ MFI } & Fast & 0.19059 & 0.076129 \\
\hline & Medium & 0.15507 & 0.076230 \\
\hline & Slow & 0.13909 & 0.068907 \\
\hline \multirow{3}{*}{ ES $r$} & Fast & 0.23384 & 0.098756 \\
\hline & Medium & 0.22511 & 0.053595 \\
\hline & Slow & 0.19817 & 0.086515 \\
\hline \multirow{3}{*}{ ESI } & Fast & 0.25544 & 0.096718 \\
\hline & Medium & 0.24975 & 0.068929 \\
\hline & Slow & 0.21288 & 0.116643 \\
\hline
\end{tabular}

(MF: Multifidus Muscles, ES: Erector Spinae muscles, r: Right, l: Left) 
Table 2. Parameter estimates (B indicate the effect of speed on each muscle).

\begin{tabular}{|c|c|c|c|}
\hline Dependent Variable & Parameter & B & SE \\
\hline \multirow{5}{*}{$\mathrm{MFr}$} & Intercept & 0.154 & 0.018 \\
\hline & Fast & 0.049 & 0.026 \\
\hline & & & \\
\hline & Medium & 0.011 & 0.026 \\
\hline & Slow & $0^{a}$ & - \\
\hline \multirow{5}{*}{ MFI } & Intercept & 0.139 & 0.017 \\
\hline & Fast & 0.052 & 0.024 \\
\hline & & & \\
\hline & Medium & 0.016 & 0.024 \\
\hline & Slow & $0^{\mathrm{a}}$ & - \\
\hline \multirow{5}{*}{ ES $r$} & Intercept & 0.198 & 0.019 \\
\hline & Fast & 0.036 & 0.027 \\
\hline & & & \\
\hline & Medium & 0.027 & 0.027 \\
\hline & Slow & $0^{\mathrm{a}}$ & - \\
\hline \multirow{5}{*}{ ESI } & Intercept & 0.213 & 0.022 \\
\hline & Fast & 0.043 & 0.031 \\
\hline & & & \\
\hline & Medium & 0.037 & 0.031 \\
\hline & Slow & $0^{\mathrm{a}}$ & - \\
\hline
\end{tabular}

a: This parameter is set to zero because it is redundant.

However in our study, the speed was not a psychological search in this field should be performed on workers who are faced with fast working conditions for a relatively long time similar to above-mentioned study. In the mentioned study, the mental aspect which can independently increase the level of muscle tension and EMG in the absence of the physical demands was studied [19-22].

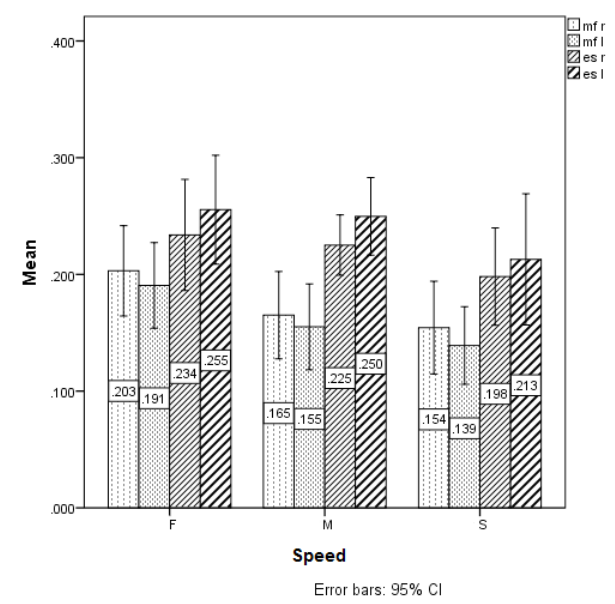

PHYSICAL TREA T MENTS

Figure 2. Muscles activity (mean value). demand but merely considered as a physical demand in a laboratory setting. Hence, as it was observed, the speed had no significant effect on the muscle activity but it had a positive effect on increasing the muscles activity levels based on the diagrams (Figure 2).

Natarajan studied the effect of lifting speed, i.e. the number of lifting per minute, in laboratory scale and observed that the speed of lifting had no effect on the stiffness of L3-L4 disk [23] that supported the results of the present work. However, the effect of lifting speed has been proven to be a risk factor for LBP [24, 25]. With regard to similar studies on the lifting speed, it can be concluded that these studies had focused on the kinematic and kinetic parameters more than EMG results [8-12].

In spite of all above-mentioned points, a few studies in the context of lifting speed effects on the lumbar muscles activity were conducted especially on the squat method. Thus, it is suggested that the studies be conducted on the health and unhealthy individuals in various skilled and unskilled population as well as on the workers group. It 
is also recommended that motion analysis be simultaneously performed with the recording the muscles activities. This study has some limitations too. It was conducted in the laboratory which had some differences with the real environments. In addition, attachment of the EMG electrodes, wires and the device to the participants reduced the sense of the natural situations.

The lifting speed is effective on the muscles activities but this effect is not significant. This result was obtained in the lab scale, thus it is possible to observe another result in natural condition. Therefore, it is recommended that other ergonomics aspects of the lifting, except the lifting speed, be considered when a person performs the lifting practice once and with light loads. However, if the person performs frequent lifting and this is his or her duty during a day, the lifting speed should be considered and the loads should be lifted slowly.

\section{Acknowledgements}

The authors are thankful to the Deputy of Research and Technology of Mazandaran University of Medical Sciences because of providing financial support for this study. Also, we are sincerely grateful to the experts of the Biomechanics Laboratory of the Ergonomics Department of University of Social Welfare and Rehabilitation Sciences and all who helped us in this study.

\section{Conflict of Interest}

The authors declared no conflict of interests.

\section{References}

[1] Smith DR, Leggat PA. Musculoskeletal disorders among rural Australian nursing students. Australian Journal of Rural Health. 2004; 12(6):241-5. doi: 10.1111/j.14401854.2004.00620.x

[2] Costa-Black KM, Loisel P, Anema JR, Pransky G. Back pain and work. Best Practice \& Research Clinical Rheumatology. 2010; 24(2):227-40. doi: 10.1016/j.berh.2009.11.007

[3] Gagnon, M. Box tilt and knee motions in manual lifting Two differential factors in expert and novice workers. Clinical Biomechanics. 1997; 12(7-8):419-28. doi: 10.1016/s02680033(97)00060-0.

[4] Gagnon M, Plamondon A, Gravel D. Pivoting with the load: An alternative for protecting the back in asymmetrical lifting. Spine. 1993; 18(11):1515-24. doi: 10.1097/00007632199318110-00017
[5] Potvin JR, Norman RW, McGill SM. Reduction in anterior shear forces on the L4L 5 disc by the lumbar musculature. Clinical Biomechanics. 1991; 6(2):88-96. doi: 10.1016/02680033(91)90005-b

[6] Toussaint HM, Van Baar CE, Van Langen PP, De Looze MP, Van Dieen JH. Coordination of the leg muscles in backlift and leglift. Journal of Biomechanics. 1992; 25(11):1279-89. doi: 10.1016/0021-9290(92)90283-7

[7] Van Dieën JH, Hoozemans MJ, Toussaint HM. Stoop or squat: A review of biomechanical studies on lifting technique. Clinical Biomechanics. 1999; 14(10):685-96. doi: 10.1016/s0268-0033(99)00031-5

[8] Hall SJ. Effect of attempted lifting speed on forces and torque exerted on the lumbar spine. Medicine and Science in Sports and Exercise. 1985; 17(4):440-4. doi: 10.1249/00005768 198508000-00006

[9] Lavender SA, Andersson GB, Schipplein OD, Fuentes HJ. The effects of initial lifting height, load magnitude, and lifting speed on the peak dynamic L5/S1 moments. International Journal of Industrial Ergonomics. 2003; 31(1):51-9. doi: 10.1016/s0169-8141(02)00174-9

[10] Davis KG, Marras WS. Assessment of the relationship between box weight and trunk kinematics: Does a reduction in box weight necessarily correspond to a decrease in spinal loading. Human Factors. 2000; 42(2):195-208. doi: 10.1518/001872000779656499

[11] Kingma I, Baten CT, Dolan P, Toussaint HM, van Dieën JH, de Looze MP, et al. Lumbar loading during lifting: A comparative study of three measurement techniques. Journal of Electromyography and Kinesiology. 2001; 11(5):337-45. doi: 10.1016/S1050-6411(01)00011-6

[12] Maab MG, Tabatabaei F. Investigating the effect of speed on the moment acting on the spine and muscles behavior during loading by utilizing motion analysis system. Paper presented at: The $6^{\text {th }}$ World Congress of Biomechanics (WCB 2010). 1-6 August 2010; Singapore, Singapore. doi: 10.1007/978-3-642-14515-5

[13] Greenland KO, Merryweather AS, Bloswick DS. The effect of lifting speed on cumulative and peak biomechanical loading for symmetric lifting tasks. Safety and health at work 2013; 4(2):105-10. doi: 10.1016/j.shaw.2013.04.001

[14] Houtman IL, Bongers PM, Smulders PG, Kompier MA. Psychosocial stressors at work and musculoskeletal problems. Scandinavian Journal of Work, Environment \& Health. 1994; 20(2):139-45. doi: 10.5271/sjweh.1419

[15] Marras WS, Mirka GA. A comprehensive evaluation of trunk response to asymmetric trunk motion. Spine. 1992; 17(3):318-26. doi: 10.1097/00007632-199203000-00013

[16] Zhang X, Xiong J, Bishop AM. Effects of load and speed on lumbar vertebral kinematics during lifting motions. Human Factors: The Journal of the Human Factors and Ergonomics Society. 2003; 45(2):296-306. doi: 10.1518/hfes.45.2.296.27242

[17] Dolan P, Adams MA. The relationship between EMG activity and extensor moment generation in the erector spinae muscles during bending and lifting activities. Journal of Biomechanics. 1993; 26(4-5):513-22. doi: 10.1016/00219290(93)90013-5 
[18] SENIAM Project. Recommendations for sensor locations on individual muscles [Internet]. 2005 [Cited 2005 Feb 13]. Available from: http://seniam.org/sensor_location.htm

[19] Lundberg U, Kadefors R, Melin B, Palmerud G, Hassmén P, Engström M, et al. Psychophysiological stress and emg activity of the trapezius muscle. International Journal of Behavioral Medicine. 1994; 1(4):354-70. doi: 10.1207/ s15327558ijbm0104_5

[20] McNulty WH, Gevirtz RN, Hubbard DR, Berkoff GM. Needle electromyographic evaluation of trigger point response to a psychological stressor. Psychophysiology. 1994; 31(3):313-6. doi: 10.1111/j.1469-8986.1994.tb02220.x

[21] Svebak S, Anjia R, Kårstad SI. Task-induced electromyographic activation in fibromyalgia subjects and controls. Scandinavian Journal of Rheumatology. 1993; 22(3):124-30. doi: $10.3109 / 03009749309099256$

[22] Wærsted, M. Attention-related muscle activity-a contributor to sustained occupational muscle load? An EMG study of the upper trapezius muscles during VDU work with minimal physical activity [PhD thesis]. Gujarat: National Institute of Occupational Health; 1997.

[23] Natarajan RN, Andersson GB. Effect of lifting speed on the biomechanical behavior of the lumbar spinal motion segment. Paper presented at: $25^{\text {th }}$ Annual Meeting of American Society of Biomechanics. 8 Aug 2001; Illinois, Chicago, USA.

[24] Greenland KO, Merryweather AS, Bloswick DS. Prediction of peak back compressive forces as a function of lifting speed and compressive forces at lift origin and destination, A pilot study. Safety and Health at Work. 2011; 2(3):236-42. doi: 10.5491/shaw.2011.2.3.236

[25] Merryweather AS, Bloswick D, Sesek R. A calculation of dynamic back compressive force: A pilot study of identify load displacement velocity constants. Journal of Safety, Health and Environmental Research. 2008; 5:1-5. 